\title{
Modélisation de la croissance en hauteur dominante du chêne sessile (Quercus petraea Liebl) en France Variabilité inter-régionale et effet de la période récente (1959-1993)
}

\author{
P Duplat*, M Tran-Ha \\ Département des recherches techniques. Office national des forêts. boulevard de Constance. \\ 77300 Fontainebleat. France
}

(Reçu le 5 mars 1996; accepté le 15 mai 1997)

\begin{abstract}
Summary - Modelling the dominant height growth of sessile oak (Quercus petraea Liebl) in France. Inter-regional variability and effect of the recent period (1959-1993). The aim is to model the dominant height growth of Quercts petraea, revealing possible effects of the region or of the recent period on the shape or level of the past growth curves of ageing stands. The data are the growth of 50 stands from five regions, 153 years old on average, reconstructed from stem analyses. A flexible mathematical model was used, with oblique asymptote the slope of which may vary with the level of the curve in the set, and with six parameters common to all curves to account for their shape and one parameter proper to each curve to account for its level (site index). The fitting, like the data themselves, exhibit a sustained growth rate till the end of observations. When analysed according to age, the residuals reveal a significant shape difference between one region and the other ones; when analysed according to date, they show an effect of the recent period, which could partially be an artefact due to the measurement method, but without any impact on our modelling of the past growth.
\end{abstract}

Quercus petraea / height growth / model / site index / global change

Résumé - L'objectif est la modélisation de la croissance en hauteur dominante du chêne sessile (Quercus petraéa Liebl) en futaie régulière, en détectant un éventuel effet régional et un éventuel effet de la période récente sur la forme ou le niveau des courbes qui décrivent la croissance antérieure de peuplements âgés. Les données utilisées sont les reconstitutions de la croissance de 50 peuplements âgés de 153 ans en moyenne, pris dans cinq régions, à partir d'analyses de tiges. On a utilisé un modèle mathématique souple, à asymptote oblique dont la pente peut varier avec le niveau de la courbe dans le faisceau, et comportant six paramètres communs à toutes les courbes pour rendre compte de leur forme et un paramètre propre à chaque courbe pour rendre compte de son niveau

\footnotetext{
* Correspondance et tirés à part

Tél : (33) 0160749210 ; fax : (3.3) 0164224973
} 
(indice de fertilité). L'ajustement, comme les données elles-mêmes, fait apparaître un accroissement soutenu jusqu'à un âge avancé. L'analyse des résidus en fonction de l'âge montre que la forme de la croissance de l'une des régions differe significativement de celle des autres. L'analyse des résidus en fonction de la date montre un effet de la période récente, qui pourrait être en partie un artefact dû aux mesures, mais qui n’a pas d'impact sur notre modélisation de la croissance passée.

\section{Quercus petraea / croissance en hauteur / modèle / indice de fertilité / changement global}

\section{INTRODUCTION}

Le chêne sessile (Quercus petraea Liebl) est un constituant majeur de la forêt des plaines et collines d'Europe, et particulièrement de France. Sa culture est d'une importance économique primordiale, de par la qualité du bois qu'il peut fournir.

Il est donc important de bien connaître ses lois de croissance, en fonction de la station où il pousse et de la sylviculture qu'il subit. En France, où il existe une vieille tradition de culture du chêne sessile de qualité en futaie régulière, cette connaissance est curieusement peu avancée, et en tous cas peu formalisée : la référence classique en la matière est une table de production pour une sylviculture unique et une fertilité unique, pour le chêne sessile de la région de la Loire (Pardé, 1962). C'est bien maigre, à une époque où l'on voudrait pouvoir mâ̂triser par la sylviculture, en fonction de la station, la croissance radiale des chênes de la récolte finale.

Le premier pas dans la connaissance des lois de croissance, et l'objectif du travail présenté ici, concerne la modélisation de la croissance en hauteur dominante, dans une situation sylvicole de référence : celle de la futaie équienne monospécifique et pas trop claire. La hauteur dominante est définie comme la hauteur moyenne des 100 plus gros arbres par hectare à chaque instant.

Dans cette situation de référence, la croissance en hauteur dominante d'une espèce dans une région est classiquement représentée par un faisceau de courbes lâge, hau- teur] croissantes et étagées. Dans ce faisceau à un paramètre libre, chaque courbe déterminée par une valeur du paramètre correspond à un niveau de «fertilité », d'autant plus élevé que la station est plus favorable à la croissance. Ce niveau est habituellement quantifié par un indice de fertilité qui est la hauteur atteinte à un âge de référence, par exemple 100 ans.

Un tel indice de fertilité, pour une espèce dans une région, varie typiquement du simple au double, voire plus, selon la station ; dans de nombreux modèles de croissance de peuplement, il sert d'indicateur du potentiel producteur de la station.

Une modélisation opérationnelle de la croissance en hauteur dominante se conçoit donc comme un système de courbes cohérentes à un paramètre libre, permettant l'expression du niveau de fertilité de chaque peuplement. Une formulation relativement générale d'un tel système pourrait être :

$$
H(A)=f(A)+b^{*} g(A)
$$

où $\mathrm{A}=$ âge $\mathrm{du}$ peuplement $\mathrm{H}=$ hauteur dominante du peuplement; $f(A)$ et $g(A)=$ deux fonctions, déterminées pour une espèce dans une région, et qui définissent la forme du faisceau; $b=$ paramètre libre, correspondant au niveau d'une courbe dans le faisceau.

Cette formulation est plus générale que la formulation strictement proportionnelle (avec $f \equiv 0$ ) qui est souvent utilisée.

En France, la grande zone de production du chêne sessile de qualité s'étend sur la 
moitié nord du pays, depuis la Normandie proche du Royaume-Uni jusqu'à l'Alsace proche de l'Allemagne. Ces deux pays voisins disposent de modèles de croissance en hauteur, qui s'appliquent au chêne sans distinction d'espèce (Forestry Commission, 1981 ; Schober, 1975 ; Zimmerle, 1930). La courbe unique de croissance en hauteur dominante de Pardé (1962) suit de très près la courbe indiquée par Schober pour la classe supérieure de fertilité. Les modèles allemands diffèrent du modèle britannique après 70 ans, âge à partir duquel ils font preuve d'une croissance plus soutenue.

Nous sommes donc amenés à nous demander si la croissance en hauteur du chêne sessile en France a une forme plus proche de celle de l'Allemagne ou de celle du Royaume-Uni, ou encore differe de l'une et de l'autre. $\mathrm{Y}$ a-t-il un gradient passant de la forme allemande en Alsace à la forme britannique en Normandie, des différences inter-régionales répondant à une toute autre logique, ou pas de différences inter-régionales au point qu'un seul modèle suffise pour toute la zone de production de qualité ? On peut aussi se demander si l'évolution récente de l'environnement, depuis les années 1950-60, a une influence importante sur la modélisation de la croissance en hauteur.

Pour répondre à ces questions, le projet suivant a été établi :

- définition de cinq régions explorant au mieux la zone de production du chêne sessile et ayant des peuplements âgés ( $>130$ ans), en futaie régulière sûre;

- choix de dix peuplements dans chaque région, couvrant au mieux la gamme des fertilités qu'on y rencontre;

- reconstitution de la croissance en hauteur dominante de chacun de ces peuplements, par analyse de tige du $1^{\mathrm{er}}, 3^{\mathrm{e}}, 5^{\mathrm{e}}$ plus gros arbre d'une placette de 6 ares, selon un protocole classique en France;

- analyse des données ainsi obtenues.

\section{MATERIEL ET METHODES}

\section{Les peuplements}

La figure 1 montre la situation géographique des cinq régions échantillonnées, à raison de dix peuplements par région. 11 a été plus difficile qu'on le pensait de trouver des peuplements quasi-purs et équiennes de chêne sessile, d'origine "futaie" sûre (régénération naturelle ou plantation), âgés et couvrant une large gamme de fertilité. Le résultat du choix effectué est illustré par le tableau I, qui en résume les principales caractéristiques région par région

La région 5, et la région 2 à un moindre degré, souffrent d'âges un peu faibles. Malgré une recherche de peuplements très poussée, la région 2 souffre d'une amplitude réduite de la fertilité, en terme de hauteur dominante à 100 ans.

Par ailleurs, cing peuplements du versant atlantique des Ardennes ont été analysés en Belgique selon un protocole similaire. Ils ne seront pas employés dans la construction des courbes de croissance en hauteur, mais seulement comparés avec les résultats issus du traitement des données françaises.

\section{Les mesures}

Selon Matérn (1976), on peut espérer avoir une estimation sans biais de la hauteur dominante (100 plus gros arbres par hectare) dans un peuplement régulier en prenant comme échantillon les $n$ plus gros arbres d'une placette de $n+1$ ares. Nous avons donc installé dans chaque peuplement retenu une placette de $600 \mathrm{~m}^{2}$, dans laquelle nous avons repéré les cinq plus gros chênes sessiles sans tenir compte de l'état de leur houppier (la seule condition est qu'ils ne soient pas dominés). Pour réduire la masse de travail sans trop perdre sur la précision, l'échantillon analysé est constitué du ${ }^{\text {er }}, 3^{\mathrm{e}}$ et $5^{\mathrm{e}}$ de ces arbres par ordre de grosseur décroissante. Les trois arbres ainsi retenus sont abattus.

Sur chacun de ces arbres, nous avons coupé des rondelles à $0,30 \mathrm{~m}$ du sol, puis à des hauteurs mesurées précisément, environ tous les $4 \mathrm{~m}$ dans la partie basse de la tige et tous les $2 \mathrm{~m}$ dans la partie haute, et mesuré la hauteur totale; nous avons compté le nombre de cernes annuels de chaque rondelle, sur la face qui correspond à la hauteur mesurée. Ce comptage a été réalisé dans 


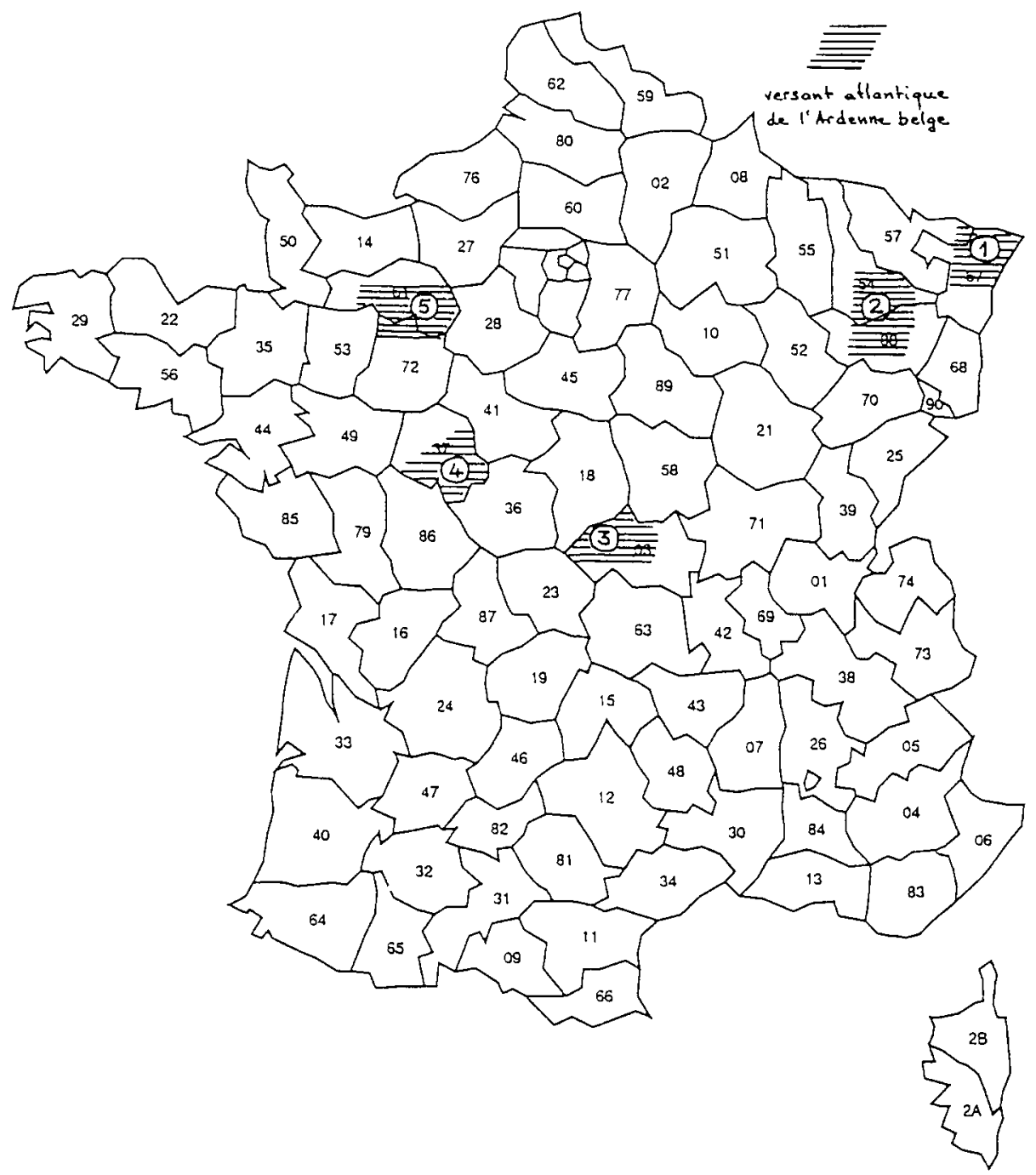

Fig 1. Carte des régions échantillonnées pour l'étude de la croissance en hauteur dominante du chêne sessile en futaie régulière.

un local électrifié, avec un bon éclairage, un rabot électrique, des épingles (pour laisser un repère tous les dix cernes), ainsi qu'un ciseau à bois bien affuté et une bonne loupe $(\times 10)$ pour les zones difficiles à lire. Chaque rondelle était lue par deux opérateurs différents, les éventuelles divergences étant résolue d'un commun accord par une lecture conjointe de la zone litigieuse.
On peut avoir une idée assez claire de la précision des mesures ainsi réalisées. En effet, la rondelle correspondant à la hauteur de $4,30 \mathrm{~m}$ de chaque arbre a été conservée et transmise au laboratoire Dynamique des systèmes forestiers (Inra-Engref) dans le cadre d'une étude sur la croissance en grosseur. Ces rondelles ont été lues au laboratoire, dans de très bonnes conditions 
Tableau I. Caractéristiques moyennes et extrêmes de l'échantillon de dix peuplements analysés dans chaque région.

\begin{tabular}{lcccccc}
\hline Région & \multicolumn{3}{c}{$\begin{array}{c}\text { Age fin } 1993 \text { (ans) } \\
\text { compté à partir de } 0,30 \mathrm{~m}\end{array}$} & \multicolumn{3}{c}{$\begin{array}{c}\text { Hauteur dominante }(\mathrm{m}) \\
\text { atteinte à l00 ans }\end{array}$} \\
\cline { 2 - 4 } & Minimum & Movenné & Maximum & Minimum & Moyenne & Maximum \\
\hline 1 & 119 & 166 & 188 & 15,1 & 23,0 & 30,7 \\
2 & 114 & 141 & 171 & 18,3 & 22,7 & 26,3 \\
3 & 102 & 152 & 186 & 18,4 & 24,7 & 29,8 \\
4 & 143 & 174 & 216 & 16,1 & 22,4 & 29,3 \\
5 & 116 & 132 & 145 & 18,8 & 24,9 & 29,2 \\
\hline
\end{tabular}

(banc de mesure spécialisé, loupe binoculaire), pour mesurer les largeurs des cernes : I'un des résultats de cette lecture était évidemment le nombre total de cernes, lu sur deux rayons opposés et relu jusqu'à avoir le même nombre sur les deux rayons ; il constitue un point de comparaison, très probablement proche de la réalité, pour notre propre mesure. L'examen de la population des écarts : $e=$ mesure $\mathrm{ONF}$ - mesure de référence Inra-Engref sur 153 rondelles ainsi doublement mesurées conduit aux résultats suivants :

$$
\begin{gathered}
\text { moyenne }(e)=-0,33 \text { an, } \\
\text { écart type }(e)=1,3 \text { an }
\end{gathered}
$$

avec $e=0$ dans $42 \%$ des cas, $e \in[-1,+1]$ dans $86 \%$ des cas, $e \in[-2,+2]$ dans $96 \%$ des cas, $e=-3$ dans 2 cas, $e=-5$ dans 3 cas, $e=-7$ dans 1 cas.

On peut donc considérer que la précision de nos mesures est très bonne, et que les reconstitutions de croissance en hauteur qu'elles permettent sont très fiables.

Il faut signaler en outre que Becker (1994), travaillant en dendrochronologie sur des carottes dans le nord-est de la France, n'a détecté aucune cerne manquant pour le chêne sessile comme pour le chêne pédonculé. Nous n'avons pas non plus détecté de cernes incomplets sur les rondelles.

En ce qui concerne les différences d'âge entre arbres d'un même peuplement, nous avons examiné l'amplitude de la fourchette (maximum-minimum) des nombres de cernes à $0,30 \mathrm{~m}$ des trois arbres de chaque placette. Elle a la distribution suivante, sur les 50 placettes échantillonnées : $1-2$ ans : 19 placettes; $3-5$ ans : 15 placettes; $6-10$ ans : 7 placettes; $11-14$ ans : 7 placettes; 31 ans : 1 placette; 34 ans : 1 placette.

Deux placettes semblent sortir des limites du raisonnable même en régénération naturelle :

-la placette 2-9, avec une fourchette de 34 ans : celle-ci est très probablement due à la présence parmi les trois arbres d'un préexistant qui a poussé pendant une trentaine d'années à l'ombre des semenciers, puis a été rejoint assez vite par les deux autres arbres ;

- la placette 5-11, avec une fourchette de 31 ans : ici les trois arbres ont trois âges différents et ne se rejoignent que plus de 50 ans après la naissance de l'arbre médian ; cette situation semble difficilement explicable dans une futaie réputée régulière.

Nous avons néanmoins conservé ces deux placettes; leurs courbes de croissance, placées dans les faisceaux de leurs régions, n'ont d’ailleurs rien de particulier.

\section{Reconstitution de la croissance en hauteur de chaque arbre}

Les emplacements des découpes, y compris la découpe à $0,30 \mathrm{~m}$, sont fixés de l'extérieur et tombent aléatoirement à l'intérieur d'une unité de croissance annuelle. Il n'en est pas de même pour le sommet de l'arbre, situé par définition à l'extrémité de la dernière unité de croissance annuelle.

L'observation de $n$ cernes à une découpe signifie que le bourgeon terminal a mis entre $n-1$ et $n$ saisons de végétations pour passer de l'emplacement de cette découpe au sommet 
actuel ; il en est de même pour la découpe à $0,30 \mathrm{~m}$. avec ses $N$ cernes.

Le bourgeon terminal a done mis, en espérance, $N-n$ saisons de végétation pour passer de $0,30 \mathrm{~m}$ à une découpe comptant $\mathrm{n}$ cernes, et $N-0.5$ saisons pour passer de $0,30 \mathrm{~m}$ au sommet actuel. L'origine des temps, pour l'arbre. étant fixée au moment où il atteint $0,30 \mathrm{~m}$, on peut ainsi reconstituer sa croissance :

- depuis le point $[0,0,30 \mathrm{~m}]$

- en passant par les points $[N-n$, hauteur de la découpe de $n$ cernes]

- jusqu' au point [ $N-0,5$, hautcur totale actuelle].

En joignant ces points par des segments de droite dans un graphique [âge, hauteur], on obtient une ligne continue que nous appelons courbe de croissance individuelle de l'arbre.

\section{Reconstitution de la croissance en hauteur dominante de chaque peuplement}

La croissance en hauteur dominante d'un peuplement est la «moyenne» des croissances individuelles en hauteur des trois arbres analysés, supposés représentatifis (même dans le passé) des 100 plus gros à l'hectare; ces trois arbres n'ont en général pas exactement le même nombre de cernes à $0.30 \mathrm{~m}$, ce qui pose un petit problème. Nous avons finalement choisi de procéder de la façon suivante:

- Nous raisonnons dans un graphique |âge, hauteur] où l'abscisse est l'âge dominant courant du peuplement

- Dans ce graphique. nous calons les trois courbes individuelles «à droite", avec leurs trois sommets sur une abscisse égale à l'âge actuel du peuplement (c'est-à-dire à la moyenne des nombres de cernes à $0,30 \mathrm{~m}$ diminuée de $0,5 \mathrm{an})$. Une ou deux de ces courbes peut ainsi «naître», à $(0,30 \mathrm{~m}$, avant l'origine des âges pour le peuplement. Après ce calage à droite, une abscisse donnée correspond à la fois à un âge donné du peuplement et à une année calendaire, la même pour les trois arbres: les hauteurs des trois courbes pour cette abscisse sont les hauteurs qu'avaient les trois arbres cette année-là (en ignorant les éventuels accidents de la cime, les petites erreurs de comptage des cernes et l'effet de l'interpolation linéaire).
Les trois courbes sont généralement assez proches : l'écart maximum observé sur toute l'étendue des âges, entre l'une quelconque des trois courbes et leur moyenne (déterminée comme il est dit ci-dessous), ne dépasse pas $2 \mathrm{~m}$ dans 40 placettes sur 50.

- La courbe moyenne part du point $[0,0,30]$ et arrive au point [âge actuel du peuplement, moyenne des hauteurs actuelles des arbres]. Entre ces deux points, elle est calculée pour chaque âge multiple de 5 ans en faisant la moyenne des trois hauteurs lues sur les trois courbes à cet instant; cette moyenne n’est réellement calculée que si les trois arbres sont déjà nés : dans le cass contraire, elle est reconstituée a posteriori par interpolation linéaire entre le premier point réellement calculé et le point $[0,0,30]$.

Ceci fait, la courbe de croissance en hauteur dominante d'un peuplement est connue, et enregistrée, au moyen d'un point [âge, hauteur] tous les 5 ans, plus un dernier point lâge actuel, hauteur actuelle dont l'âge n'est généralement pas un multiple de 5 ans. L ensemble des 50 courbes «observées» ainsi obtenues ( 10 par région), totallisant 1608 points, constitue nos données. Nous les avons dessinées, région par région, sur cinq graphiques |âge, hauteur dominantel à la même échelle (fig 2).

Pour avoir une idée plus précise d'éventuelles différences de forme entre les régions, notamment dans la phase juvénile de croissance rapide. nous avons aussi dessiné sur un même graphique la moyenne des dix courbes de chaque région pour les âges ò̀ ces dix courbes existent (jusqu ‘à 102-143 ans selon les régions).

\section{Le modèle mathématique}

Étant donné un ensemble de courbes de croissance en hauteur observées, nous lui ajustons le modèle mathématique suivant :

$$
\mathrm{H}=0,30+\left(\mathrm{a} * \mathrm{~A}+\mathrm{b}_{i}|*| \mathrm{l}-\mathrm{e}^{-\left.\mathrm{A}^{\mathrm{A}}{ }^{\mathrm{j}}\right|^{\mathrm{r}}}+\mathrm{p}+\mathrm{m} * \mathrm{~b}_{i} * \mathrm{~A}\right.
$$

[modèle MM]

où A est l'âge dominant, compté à partir du moment où la hauteur dominante atteint $0,30 \mathrm{~m}$ : $\mathrm{H}$ est la hauteur dominante; e est la base du logarithme népérien; $\mathrm{a}, \mathrm{c}, \mathrm{d}, \mathrm{r}, \mathrm{p}, \mathrm{m}$ sont des paramètres communs à toutes les courbes de l'ensemble, et rendent compte de la forme du 

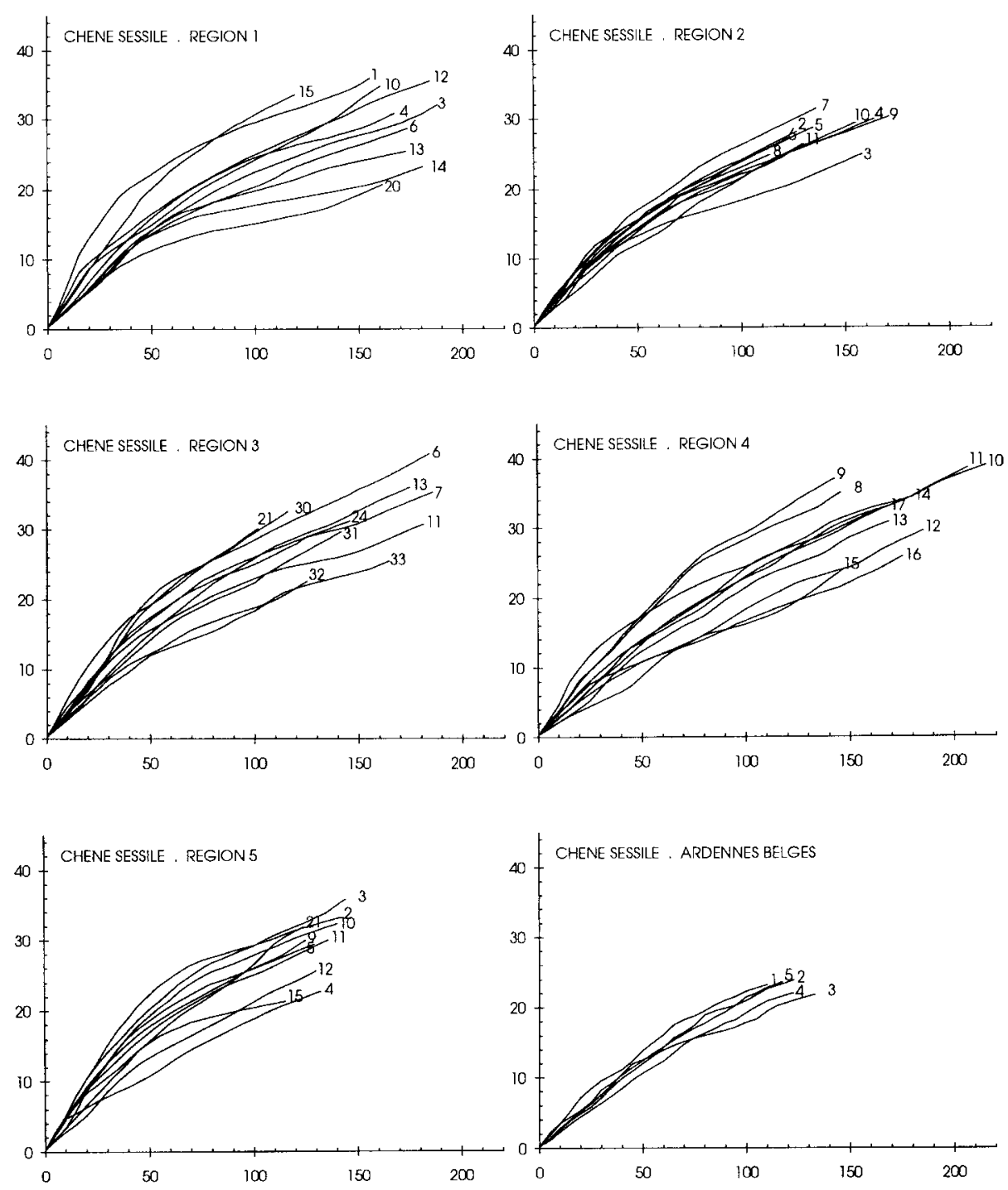

Fig 2. Dessin des données observées : courbes de croissance en hauteur dominante de dix peuplements dans cinq régions françaises et de cinq peuplements dans une région belge (abscisse : âge dominant compté à partir de $0,30 \mathrm{~m}$ en années, ordonnée : hauteur dominante en mètre).

faisceau ; m est contraint à être positif ou nul; $b_{i}$ est un paramètre dont la valeur est propre à chaque courbe $i$ de l'ensemble, et rend compte de son niveau dans le faisceau, ce qui lui donne le statut d'un indice de fertilité quand les autres paramètres sont fixés.
Le modèle MM entre dans la catégorie générale formulée en introduction. Il présente des asymptotes obliques, ayant un point commun de coordonnées $\mid-1 / \mathrm{m},-(\mathrm{a}+\mathrm{p}) / \mathrm{m}]$ et dont la pente $a+p+m^{*} b_{i}$ est linéairement croissante avec le niveau de la courbe dans le faisceau. Ceci est en 
accord avec l'allure générale des données et admet en cas particulier la modélisation d'asymptotes parallèles $(m=0)$.

Ce modèle, très souple, a été utilisé avec succès pour décrire la croissance en hauteur dominante de diverses espèces en futaie régulière ou en taillis simple (Duplat et Tran-Ha, 1990). En contre-partie, il comporte six paramètres de forme et un paramètre de niveau ; les estimations des six paramètres de forme sur un ensemble de données sont forcément très corrélées : il ne faut pas chercher à les interpréter directement; il ne faut pas non plus traduire la question de différence de forme entre plusieurs ensembles de courbes en question de différence significative entre paramètres homologues.

Chaque courbe des données est décrite comme une suite de points, à raison d'un point tous les 5 ans plus un dernier point correspondant à l'âge et à la hauteur dominants actuels. L'ajustement du modèle, non linéaire, sur un ensemble de courbes est fait à partir de tous les points qui les décrivent, en utilisant des variables muettes pour relier chaque point à sa courbe (tous les points d'une courbe $i$ ont en commun la même valeur $b_{i} d u$ paramètre de niveau). Formellement, dans l'expression du modèle mathématique à ajuster, on remplace $\ll b_{i} »$ par $\ll b_{1}{ }^{*} X_{1}+b_{2}{ }^{*} X_{2}+\ldots+$ $\mathrm{b}_{i}{ }^{*} \mathrm{X}_{i}+\ldots »$, la variable muette $\mathrm{X}_{i}$ prenant la valeur 1 pour tous les points de la courbe $i$, et la valeur 0 pour les autres points (Duplat et TranHa, 1990).

Notre programme de régression non linéaire est basé sur une méthode itérative de gradient proposée par Schmidt (1982), nécessitant l'explicitation des dérivées partielles du modèle par rapport aux paramètres. Il demande une valeur initiale des paramètres et s'arrête à la première itération qui satisfait l'un des critères de convergence (deux estimations successives quasi-semblables de tous les paramètres ou quasi-invariance de la somme des carrés des écarts résiduels), en fournissant :

- les estimations $\hat{a}, \hat{c}, \hat{d}, \hat{r}, \hat{p}, \hat{m}$ des paramètres communs, qui entrent dans l'expression finale du modèle ajusté;

- les estimations $\hat{b}_{i}$ du paramètre propre à chaque courbe, qui permettent de déterminer les résidus (modèle ajusté - observation) ;

-le résidu quadratique moyen s.

Dans l'expression finale d'un tel modèle, â, ĉ, $\hat{\mathrm{d}}, \hat{\mathrm{r}}, \hat{\mathrm{p}}$, m étant fixés, on peut faire figurer un indice de fertilité plus habituel que $b_{i}$ : la hau- teur $\mathrm{H}_{r e f i}$ atteinte à un âge de référence $\mathrm{A}_{r e f}$; il suffit $\mathrm{d}$ y remplacer $b_{i}$ par son expression formelle en fonction de $\mathrm{A}_{r e f}$ (qui a une valeur fixée, par exemple 100 ans) et $\mathrm{H}_{\text {ref }}$, obtenue en résolvant l'équation linéaire en $b_{i}$ qui correspond à la condition «la courbe de niveau $\mathrm{b}_{i}$ passe par le point $\left[\mathrm{A}_{r e f}, \mathrm{H}_{\text {refi } i}\right] \gg$ :

$$
\mathrm{H}_{r e f i}=\mathrm{H}\left(\mathrm{A}_{r e f}, \hat{\mathrm{a}}, \hat{\mathrm{c}}, \hat{\mathrm{d}}, \hat{\mathrm{r}}, \hat{\mathrm{p}}, \hat{\mathrm{m}}, \mathrm{b}_{i}\right)
$$

C'est ce que nous avons fait pour les modèles finalement retenus au paragraphe «Discussion et conclusion. Variabilité inter-régionale de la forme de la croissance».

\section{Ajustements}

Nous avons ajusté le modèle séparément à chacune des cinq régions, obtenant pour chacune une estimation de a, c, d, r, p, $m$ et dix estimations de $b_{i}$ (une par courbe). Le résidu quadratique moyen ainsi obtenu dans une région quantifie la variabilité irréductible des courbes à l'intérieur de cette région, autour d'un schéma tel que le nôtre avec un seul paramètre libre $\left(b_{i}\right)$ pour rendre compte du niveau de chaque courbe dans le faisceau (indice de fertilité). Ce schéma est correct s`il n'y a pas de relation, à aucun âge, entre les résidus et le niveau. La variabilité en question est alors une variabilité de forme autour de la forme-type définie par a, c, d, r, p, m, et non une variabilité de niveau, puisque celui-ci est explicité par le $b_{i}$ propre à chaque courbe.

Le résidu quadratique moyen sur l'ensemble des cinq régions est un indicateur de précision globale de ce modèle fin (modèle A) composé de cinq sous-modèles (modèles $\mathrm{A}[1], \mathrm{A}[2], \mathrm{A}[3]$, $\mathrm{A}[4], \mathrm{A}[5]$ respectivement établis sur et appliqués aux régions $1,2,3,4,5$ ).

Nous avons ensuite ajusté le modèle sur l'ensemble des cinq régions ( 50 courbes), obtenant une estimation de a, c, d, r, p, m et 50 estimations de $b_{i}$ (une par courbe). Le résidu quadratique moyen est un indicateur de précision globale de ce modèle grossier unique (modèle $\mathrm{B}$, établi sur l'ensemble des régions). Le résidu quadratique moyen à l'intérieur d'une région est un indicateur, quand on le compare à celui du modèle A dans la même région, de la perte de précision entraînée par l'usage du modèle unique $\mathrm{B}$ pour cette région.

D'une façon analogue au modèle A, nous avons ajusté des modèles « mi-fins » constitués de 
deux ou trois sous-modèles ajustés chacun sur un sous-ensemble des régions : à partir des dissemblances et ressemblances suggérées par l'examen visuel, et de l'étude des résidus du modèle d'ensemble B, nous avons essayé les modèles suivants :

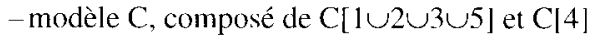

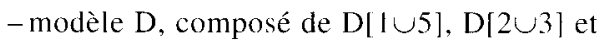
D[4]

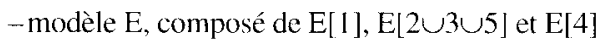

Les sous-modèles C[4], D[4], E[4] sont le sous-modèle A[4]; E[1] est A[1].

Ces différents modèles sont «emboités », au sens où ils sont constitués de sous-modèles bâtis sur et pour des partitions elles-mêmes embô̂tées. On peut ainsi décrire deux hiérarchies, en allant du plus fin au plus grossier : $A \subset D \subset C \subset B$ et $\mathrm{A} \subset \mathrm{E} \subset \mathrm{C} \subset \mathrm{B}$; D et $\mathrm{E}$ ne sont pas emboîtés.

Pour tester, dans une hiérarchie, si un modèle plus grossier est significativement moins bon que le modèle fin, on pourrait penser à utiliser le test suivant (Seber et Wild, 1989):

$$
\mathrm{F}_{\text {cal }}=\begin{gathered}
\mathrm{SCE}_{g r o s}-\mathrm{SCE}_{f i n} \\
\mathrm{p}_{f i n}-\mathrm{p}_{g r o s}
\end{gathered} / \begin{aligned}
& \mathrm{SCE}_{f i n} \\
& n-\mathrm{p}_{f i n}
\end{aligned}
$$

avec $\mathrm{SCE}_{\text {gros }}, \mathrm{SCE}_{\text {fin }}=$ somme des carrés des résidus du modèle grossier, du modèle finn ; $\mathrm{p}_{g g \text { ros' }}$, $\mathrm{p}_{f i n}=$ nombre de paramètres du modèle grossier, du modèle fin: $n=$ nombre de données (points âge $\times$ hauteur) utilisées pour l'ajustement $F_{\text {calc }}$ tendant asymptotiquement vers un $\mathrm{F}$ de Snedecor...

En fait, un tel test n'est probablement pas utilisable ici, à cause de la nature des données: elles sont constituées de points successifs serrés sur un petit nombre de courbes, et l'hypothèse d'indépendance des résidus ne peut absolument pas être considérée comme vérifiée (auto-corrélation positive sur une courbe). Encore plus que la non-normalité éventuelle des résidus, ceci interdit d'admettre que les deux numérateurs dans $\mathrm{F}_{\text {calc }}$ sont des $\chi^{2}$. Nous considérons donc les résidus quadratiques moyens uniquement comme des indicateurs de précision de l'ajustement, sans faire sur eux de tests statisiques. C'est plutôt à une analyse directe des résidus euxmêmes que nous demanderons une réponse à la question d'une éventuelle différence significative de forme entre régions.

\section{Analyse des résidus}

Pour chaque modèle ajusté, et pour chaque point (âge tous les 5 ans) de chaque courbe, nous avons un résidu $=$ (valeur donnée par le modèle - valeur observée). Le nuage de points [âge, résidu] d'une région, les points issus d'une même courbe étant reliés par un trait, permet une première détection d'éventuelles anomalies, notamment celle de tendances particulières des résidus selon l'âge ou la fertilité.

Mais c'est surtout la moyenne des résidus dans une région, à âge donné, qui nous intéresse: si, pour un modèle commun à plusieurs régions, cette moyenne est significativement différente de 0 , de façon consistante sur certaines tranches d'âge, on peut dire que cette région présente en fait une croissance de forme différente de celle dont le modèle commun rend compte.

À âge fixé, on peut faire des tests statistiques sur les résidus : leur indépendance entre courbes pour un même âge peut facilement être admise. Nous avons utilisé systématiquement trois tests, aux seuils de 5 et $10 \%$ :

- le test de Student, qui suppose que la distribution des résidus est normale;

- le test non-paramétrique des signes et rangs de Wilcoxon, qui suppose seulement cette distribution symétrique;

- le test non-paramétrique du signe, qui ne suppose plus rien, mais porte en fait sur la médiane.

Indépendemment d'éventuelles différences de forme, nous avons aussi voulu visualiser un éventuel effet de la date sur les résidus. Nous avons donc calculé, pour chaque courbe :

- les âges correspondant à 1993, 1993 - 5, 1993 $10,1993-15 \ldots$ etc ;

- les hauteurs observées correspondantes, par interpolation:

- les hauteurs modélisées correspondantes, à partir du modèle ajusté le plus fin, A ;

- les moyennes, par région et par date, des résidus ainsi obtenus.

\section{RÉSULTATS}

\section{Examen visuel}

La vue des cinq faisceaux de courbes (fig 2) suggère quelques commentaires : 
- Naturellement, le faisceau de chaque région n'est pas constitué de courbes aussi harmonieusement étagées que dans les modèles. Les croisements sont assez nombreux avant 50 ans, ce qui peut être lié en partie à la variabilité de la durée de la régénération naturelle (enlèvement des adultes par coupes progressives). En outre, certaines courbes ont une forme de croissance différente de l'ensemble des autres courbes de la même région (voir notamment les peuplements 15 et 21 de la région 5 ).

- La forme de la croissance jusqu'à 100 ans semble plus tendue dans la région 4 , et un peu moins tendue dans la région 1 , que dans les régions $2,3,5$.

- La croissance au stade adulte semble se prolonger indéfiniment (dans la limite des âges observés) à un rythme régulier ; ce rythme peut paraître indépendant de la fertilité comme dans la région 4 , ou dépendant de la fertilité comme dans la région 1 . Ceci justifie l'adoption d'un modèle mathématique à asymptotes obliques, de pente constante ou croissante avec la fertilité. On peut même dire que cela disqualifie l'emploi de modèles à asymptotes horizontales.
- Un certain nombre de courbes présentent une accélération de la croissance dans les dernières décennies, précédée ou non d'un ralentissement : voir par exemple les peuplements 4 et 10 de la région 1 . Ce relèvement final des courbes n'est pas général, mais assez fréquent, alors qu'on observe plus rarement un fléchissement final.

L'examen des courbes juvéniles moyennes par région (fig 3 ) permet de confirmer et de préciser le second commentaire ci-dessus : les régions 2 et 3 ont une forme moyenne semblable ; la région 4 a une forme plus tendue, et la région 1 une forme moins tendue ; la région 5 présente une forme intermédiaire entre la région 1 et le couple 2, 3. Ces constatations ont conduit à tester les différents modèles avec sous-modèles présentés dans la section «Ajustements».

\section{Résultat des ajustements}

Les estimateurs obtenus pour les paramètres $\hat{a}, \hat{c}, \hat{d}, \hat{r}, \hat{p}, \hat{m}$ de chacun des modèles sont donnés dans le tableau II. Les $\hat{b}$, propres à

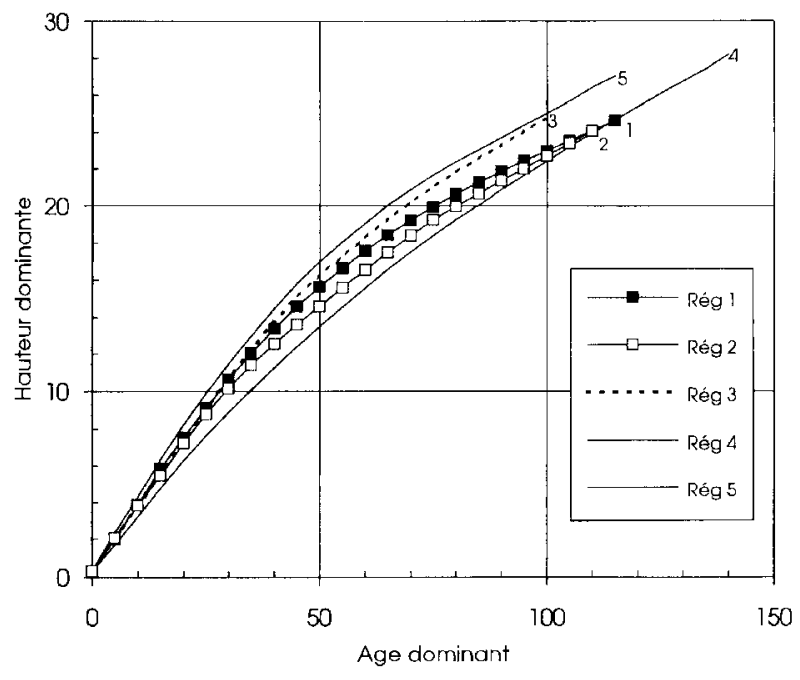

Fig 3. Moyenne des courbes observées dans chaque région ; cette moyenne est calculée sur les dix courbes de la région, pour les âges où ces dix courbes existent (abscisse : âge dominant compté à partir de $0,30 \mathrm{~m}$ en années, ordonnée : hauteur dominante en mètre). 


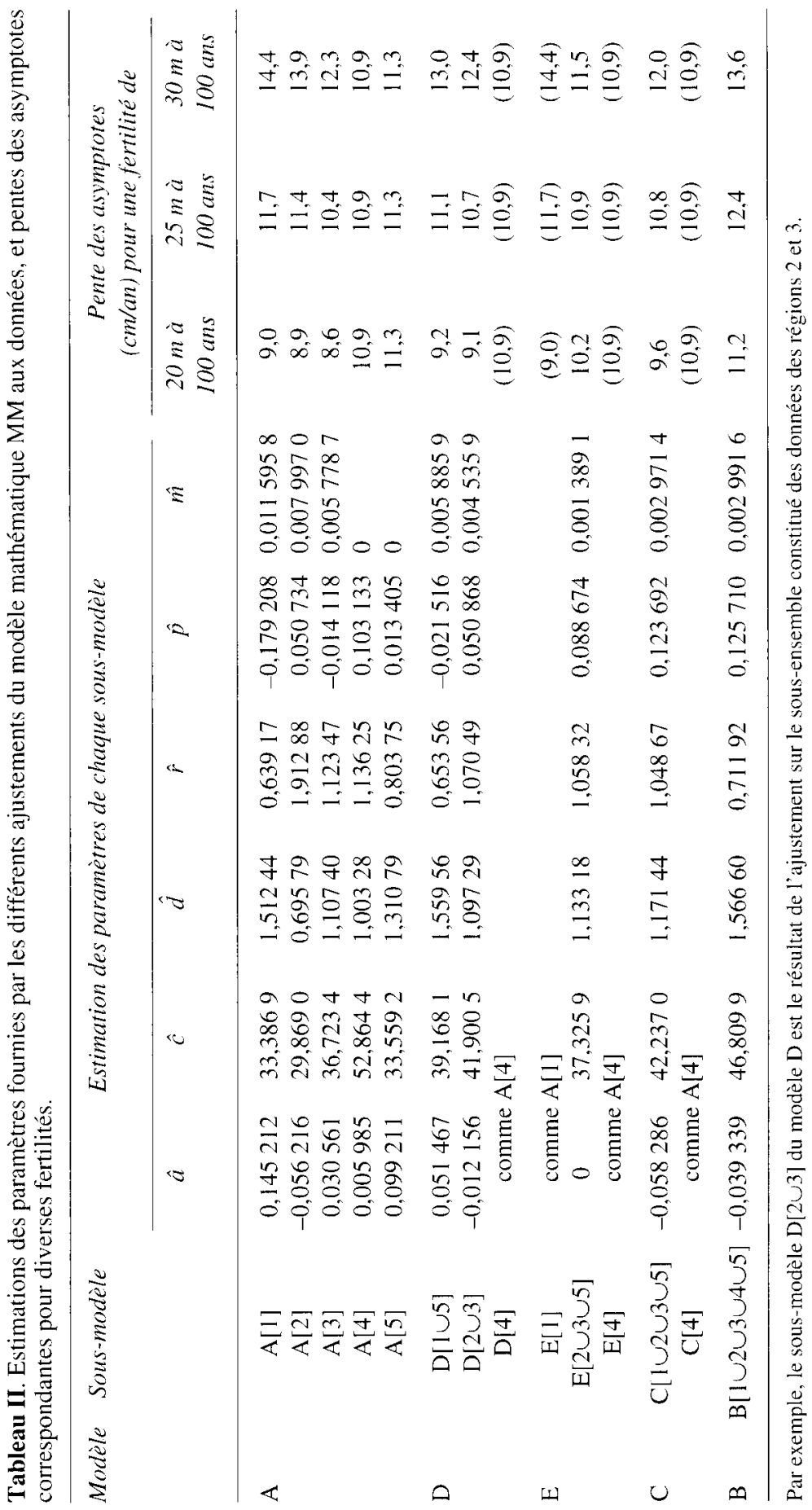


chaque courbe, qui ne servent qu'au calcul des résidus et n'entrent pas dans l'expression finale des modèles, n'y figurent pas. On y constate en particulier que les sous-modèles ajustés sur la région 4 et sur la région 5 , $\mathrm{A}[4]$ et $\mathrm{A}[5]$, comportent des asymptotes parallèles ( $\hat{\mathrm{m}}=0$ ), ce qui est cohérent avec l'impression visuelle procurée par la figure 2. La figure 7 montre un exemple des courbes fournies par l'ajustement, pour les modèles $\mathrm{B}$ et $\mathrm{C}$.

L'estimation de chaque paramètre est très peu robuste, mais celle de la pente des asymptotes (c'est-à-dire $\hat{\mathbf{a}}+\hat{\mathrm{p}}+\hat{\mathrm{m}} * \mathrm{~b}_{i}$ pour la courbe dont le niveau est caractérisé par $b_{i}$ ) l'est probablement beaucoup plus. Nous avons donc calculé cette pente, pour des valeurs de $b_{i}$ correspondant dans chaque modèle à des fertilités médiocre, moyenne et bonne caractérisées par 20, 25, 30 m de hauteur dominante à 100 ans (tableau II). Pour une fertilité moyenne, les estimations fournies par les différents ajustements sont bien consistantes, entre modèles et entre régions ou sous-ensembles de régions, et de l'ordre de $11 \mathrm{~cm} / \mathrm{an}$. La variation de pente avec la fertilité, dans les régions où l'ajustement en fait apparaître une, n'est pas négligeable: 4 ou $5 \mathrm{~cm} / \mathrm{an}$ quand l'indice de fertilité passe de 20 à $30 \mathrm{~m}$ (à 100 ans).

En termes d'écart résiduel quadratique moyen, les résultats sont résumés dans le tableau III.

En accord avec le premier commentaire suggéré par l'examen visuel des courbes observées, l'écart résiduel intra-région irré-

Tableau III. Résidus quadratiques moyens (en mètre) résultant des ajustements qui conduisent aux modèles A, D, E, C, B.

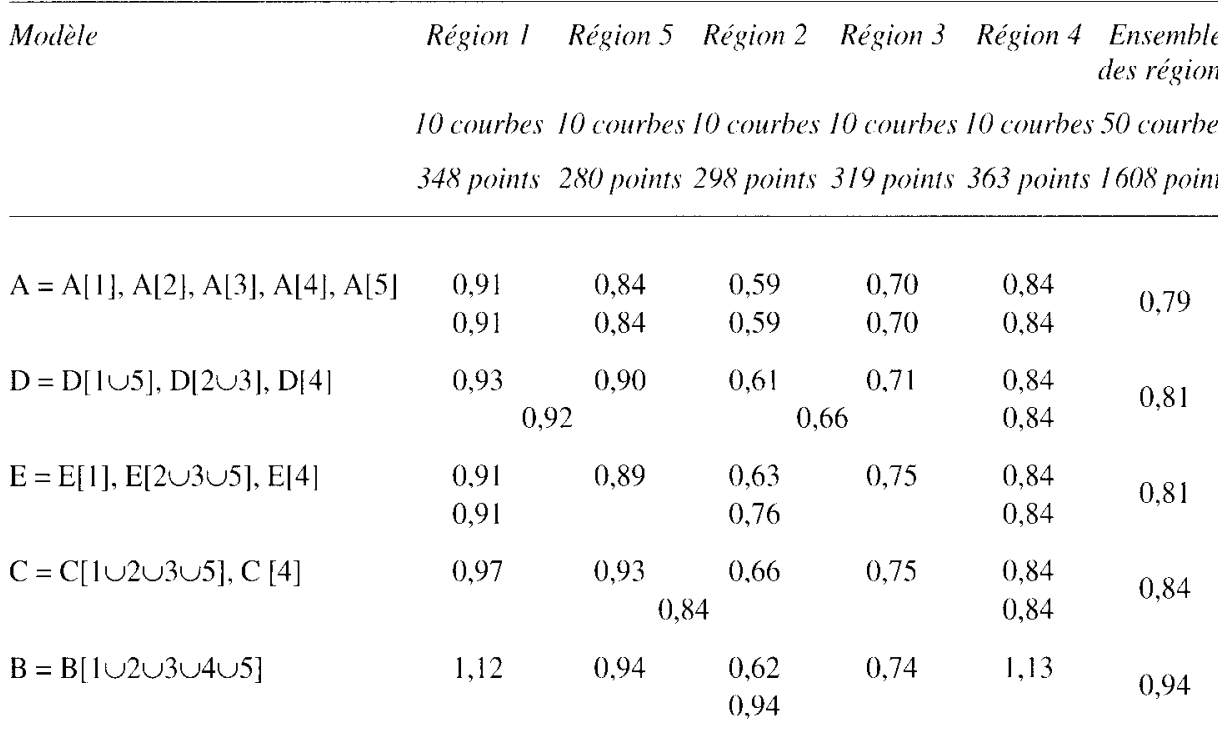

Pour chaque modèle, la première ligne donne le résidu intra-région, par application du sous-modèle pertinent aux données de la région; la deuxième ligne donne le résidu intra-sous-ensemble (=regroupement des régions concernées par un même sous-modèle), par application du sous-modèle pertinent aux données du sous-ensemble; la dernière colonne donne le résidu du modèlc sur l'ensemble des données, par application aux données de chaque région du sous-modèle pertinent. Par exemple : A[3] est le sous-modèle pertinent pour la région 3 dans le modèle A ; $\mathrm{D}[2 \cup 3]$ est le sous-modèle pertinent pour la région $3 \mathrm{et}$ pour le sous-ensemble [2 3 ] dans le modèle D, etc. 
ductible est assez élevé (de $0,59 \mathrm{~m}$ pour la région 2 à $0,91 \mathrm{~m}$ pour la région 1 ); rappelons que sa valeur n'est pas liée à la variabilité de niveau des courbes dans la région (absorbée par l'explicitation du $\hat{b}$, propre à chaque courbe dans le calcul des résidus), mais à la variabilité de leur forme à niveau donné.

Du point de vue de l'écart résiduel global sur l'ensemble des cinq régions, les modèles se classent évidemment dans l'ordre attendu, du plus fin (A : $0,79 \mathrm{~m})$ au plus grossier (B : $0,94 \mathrm{~m}$ ). Les performances de D et $\mathrm{E}$, constitués chacun de trois sous-modèles, sont équivalentes et peu différentes de celle du modèle le plus fin, $A$.

Les modèles $D$ et $E$ diffèrent l'un de l'autre par le regroupement de la région 5 : avec la région 1 dans $\mathrm{D}$, avec les régions 2 et 3 dans E; la perte de précision qui s'ensuit pour elle est équivalente dans les deux cas, mais les régions 2 et 3 perdent un peu plus à l'usage du modèle $\mathrm{E}$ qu'à celui du modèle D. L'examen de la moyenne par région des résidus à âge donné est aussi en faveur du modèle D (voir fig 5). C'est donc le modèle
$\mathrm{D}$, et la hiérarchie $\mathrm{A} \subset \mathrm{D} \subset \mathrm{C} \subset \mathrm{B}$, que nous retiendrons en cas de besoin.

On peut remarquer que la performance globale du modèle $C$, qui explicite les particularités de la région 4 en lui consacrant un sous-modèle, est nettement meilleure que celle du modèle d'ensemble B. Il convient néanmoins de noter que le modèle $\mathrm{B}$, malgré sa "grossièreté", explique quand même $99 \%$ de la variation de hauteur exprimée dans le nuage des 1608 points [âge, hauteur] que constituent les données $\left(\mathrm{R}^{2}=\right.$ $0.990)$.

\section{Résultats de l'analyse des résidus}

Pour le modèle $\mathrm{A}$, dans chaque région, nous avons examiné les dix courbes de résidus en fonction de l'âge ; la figure 4 en montre un exemple, pour la région 1 . On y voit bien l'auto-corrélation temporelle positive entre résidus successifs d'une même courbe : les courbes de résidus sont bien régulières et $80 \%$ d'entre elles ne recoupent pas l'axe des zéros plus de trois fois, alors qu'elles

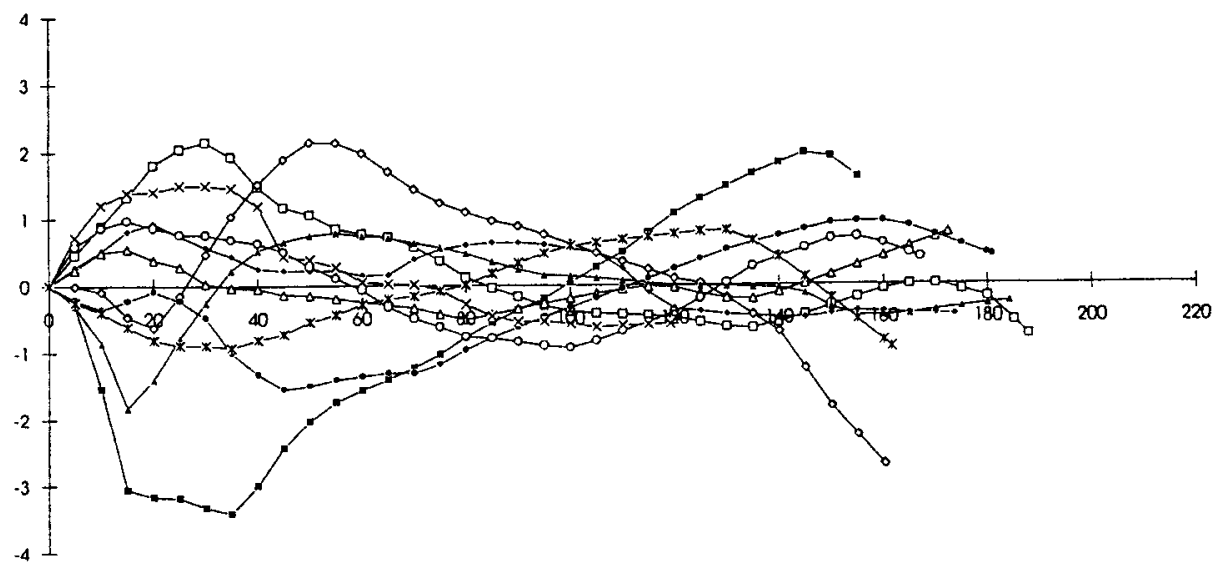

Fig 4. Résidu de la courbe de croissance ajustée par le modèle A de chaque peuplement, par rapport à sa courbe de croissance observée, en fonction de l'âge. Exemple des dix peuplements de la région 1 (sous-modèle $\mathrm{A}[1]$ ). Résidu = valeur fournie par le modèle - valeur observée (abscisse : âge en années, ordonnée : résidu en $\mathrm{m}$ ). 

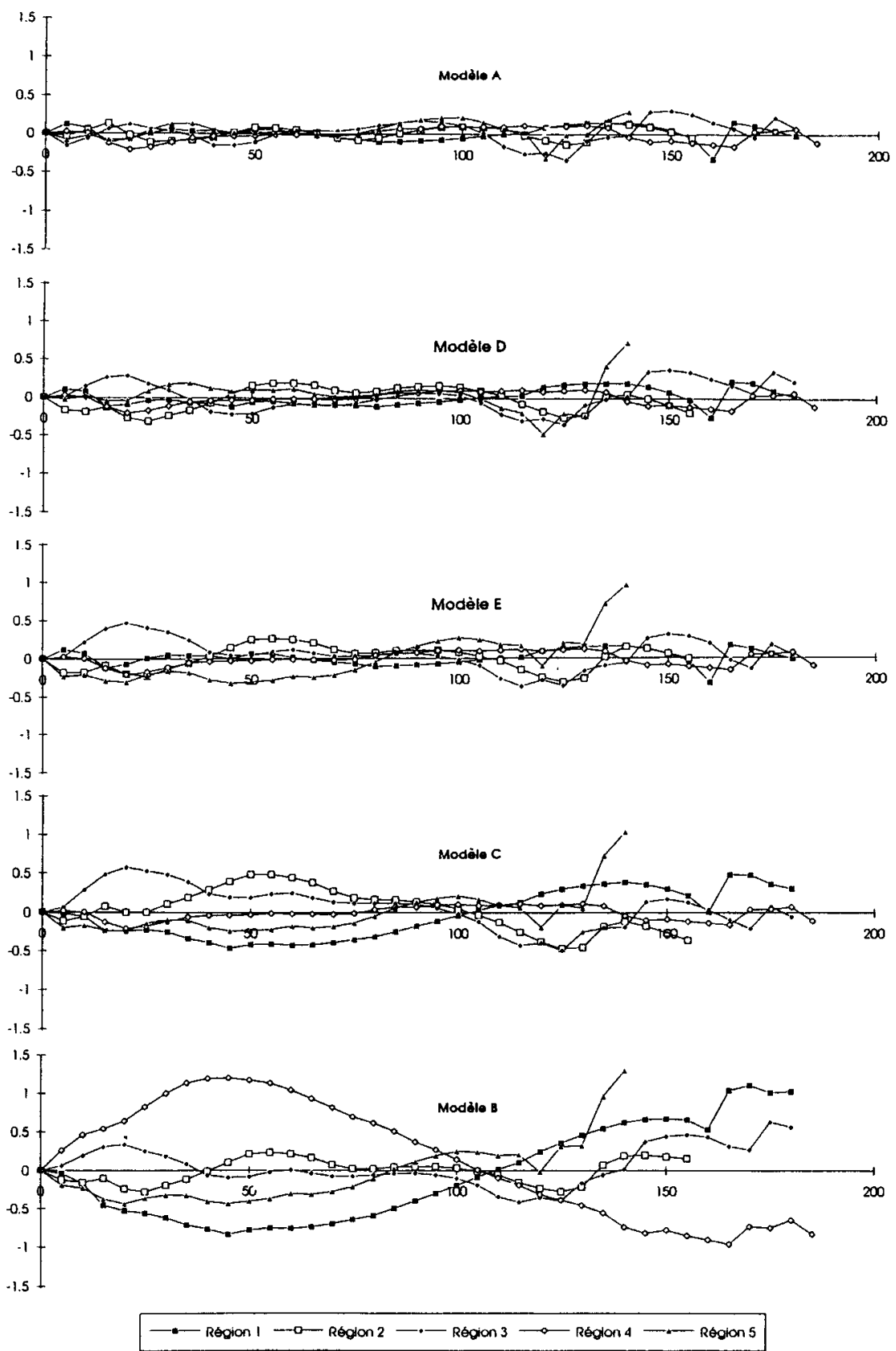

Fig 5. Moyenne des résidus de chaque région à âge donné pour les modèles A, D, E, C, B (abscisse : âge en années,ordonnée : résidu moyen en mètres). Chaque moyenne régionale est calculée sur les courbes existantes $(\leq 10)$ à l'âge considéré, tant que leur nombre est supérieur ou égal à 3. 
sont constituées d'une trentaine de points. En revanche, on n'y détecte dans aucune région de tendance particulière en fonction de l'âge et de la fertilité, ce qui confirme la pertinence du modèle mathématique MM pour ce problème.

Considérons maintenant la moyenne des résidus à âge donné dans chaque région. $\mathrm{La}$ figure 5 représente ces moyennes régionales pour les cinq modèles testés A, D, E, C, B. Sa lecture est très parlante :

- modèle B : ce modèle unique, s'il est sans biais pour l'ensemble des régions, ne convient pas bien pour les régions 1 et 4 ; il est trop tendu pour la région 1 (résidus d'abord négatifs, puis positifs), et pas assez pour la région 4 (l'inverse).

- modèle $\mathrm{C}$ : avec ce modèle qui explicite deux sous-ensembles $(1 \cup 2 \cup 3 \cup 5,4)$ la situation s'améliore; la région 4 ne pose plus de problème puisque le modèle $\mathrm{C}$ en rend compte par un sous-modèle particulier; très logiquement, la région 1 est nettement mieux représentée que par le modèle $\mathrm{B}$, la région 5 a un peu gagné au change, les régions 2 et 3 y ont un peu perdu.

-modèle D : avec ce modèle qui explicite trois sous-ensembles $(1 \cup 5,2 \cup 3,4)$, le progrès est encore net et l'on peut considérer qu'aucune région ne pose de problème particulier.

- modèle A : c'est la référence, chaque région y ayant son sous-modèle particulier.

Venons-en maintenant à la question des éventuelles différences significatives de forme entre régions, en la formulant de façon un peu plus pragmatique : étant donné un modèle $B, C$, ou $D$, existe- $t-1$ des régions dont la forme de croissance diffère significativement de celle du modèle ?

Pour une région, la réponse sera positive si le résidu moyen à âge donné est significativement différent de zéro de façon consistante sur une ou plusieurs tranches d'âge. Chaque résidu moyen est soumis à trois tests, comme il est dit dans la section Ana- lyse des résidus. Le tableau IV en donne les résultats pour les modèles $\mathrm{B}, \mathrm{C}$, et D (il n'y a aucune différence significative pour le modèle $\mathrm{A}$, soumis aux mêmes tests à titre de vérification).

Ces résultats sont assez clairs :

- Modèle B : la forme du modèle unique ne convient manifestement pas à la région 4 , et pas très bien à la région 1 ; on risque, en l'utilisant pour ces régions, un biais de l'ordre de $1 \mathrm{~m}$ dans un sens autour de 50 ans, puis dans l'autre autour de 150 ans (voir fig 5). La région 3 est moins préoccupante: significativité d'un ou deux tests sur une tranche d'âge étroite, liée à un biais assez faible (inférieur à $0,40 \mathrm{~m}$ ) mais affecté d'un écart type encore plus faible $(0,15 \mathrm{~m})$. Les régions 2 et 5 ne diffèrent pas significativement de la forme du modèle unique.

-Modèle C : l'amélioration obtenue en distinguant la région 4 des autres est très sensible ; la région 3 pose le même petit prolème qu'avec le modèle $B$; celui que pourrait poser la région 2 semble négligeable vu l'ampleur du biais en jeu (inférieur à $0,20 \mathrm{~m}$, entre 75 et 90 ans); les régions 1, 5, 4 ne présentent aucune différence significative avec le modèle, c'est-à-dire avec les sous-modèles $C[1 \cup 2 \cup 3 \cup 5]$ et $C[4]$ respectivement.

- Modèle D : la distinction en trois sousensembles ne conduit à un biais significatif que sur une tranche de 10 ans dans une seule région, et son ampleur est négligeable $(0,15 \mathrm{~m})$.

\section{Effet de la date}

Nous l'avons étudié à partir des résidus du modèle A qui rend le mieux compte, région par région, des effets de l'âge et de la fertilité, et qui est donc le plus pertinent pour les éliminer. Le résultat en est illustré par la figure 6, qui fait apparaître une tendance commune : depuis 1960 environ, on observe une «descente» des résidus moyens, c'est- 


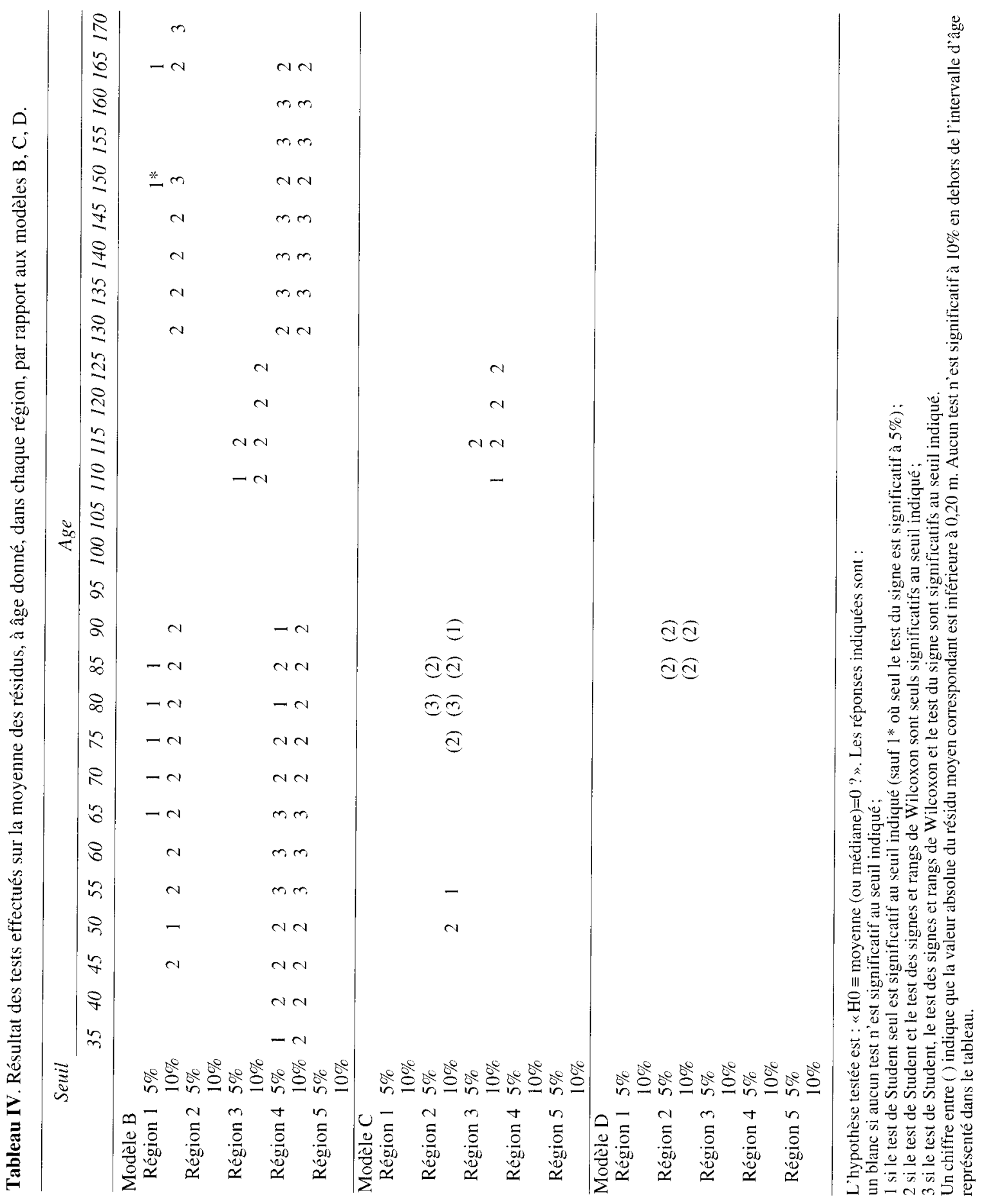


Résldu moyen por région d date donné, par ropport ou modèle A
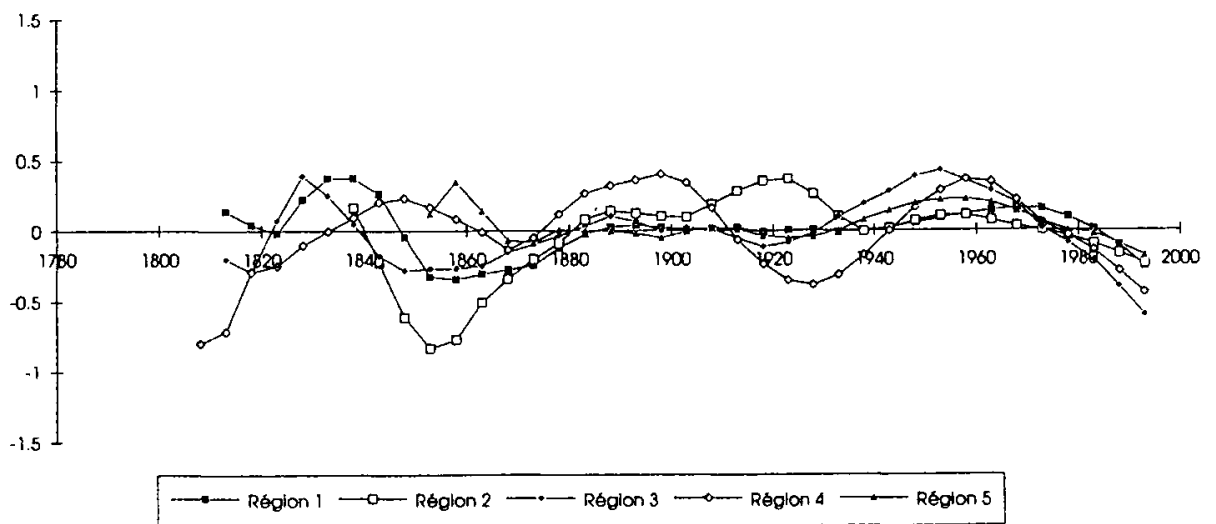

Fig 6. Moyenne sur chaque région des résidus à date donnée. en fonction de la date, pour le modèle A (date en abscisse, résidu moyen en mètres en ordonnée). Chaque moyenne régionale est calculée sur les courbes existantes à la date considérée, tant que leur nombre est supérieur ou égal à 3.

à-dire une remontée des courbes par rapport à leurs sous-modèles respectifs, plus ou moins précédée suivant les régions d'une variation dans l'autre sens. Cette tendance est nettement plus marquée pour les régions 3 et 4 (amplitude de l'ordre de $\pm 0,50 \mathrm{~m}$ ) que pour les autres $( \pm 0,20 \mathrm{~m})$.

On observe aussi des fluctuations avant 1940, d’amplitude équivalente, supéricure à celles des résidus moyens en fonction de l'âge, mais sans synchronisation claire entre les régions. Pendant la période 1880-1930, ceci ne concerne que les régions 2 et 4 , les trois autres étant très «calmes".

\section{Étude des données belges}

Les cinq courbes observées sur le versant atlantique des Ardennes, par H. Claessens et A. Thibaut (Irsia, faculté des sciences agronomiques de Gembloux) sont dessinées avec les courbes françaises dans la figure 2. Il faut noter qu'elles couvrent une gamme de fertilité très étroite, s'arrêtent vers 120 ans et sont peu nombreuses : autant de raisons d'être prudent et ne pas généraliser trop vite les résultats que nous pourrons en tirer. Nous les avons confrontées aux modèles obtenus en France, pour savoir de quelle région elles se rapprochent le plus et de quels modèles elles peuvent s'accomoder. Un simple examen visuel révèle une certaine ressemblance de forme avec la région 4.

Les données sont mises sous la même forme que les données françaises. On y a alors ajusté chacun des sous-modèles obtenus en France, avec la valeur correspondante de leurs paramètres $a, c, d, r, p, m$ déjà fixée (valeurs indiquées dans le tableau II) : l'ajustement ne sert qu'à estimer le $\hat{b}$, propre à chacune des cinq courbes, dans le cadre de la forme décrite par chacun des sousmodèles. Cela permet de calculer les résidus de chaque sous-modèle par rapport à ces données nouvelles, et en particulier son résidu quadratique moyen qui est un bon indicateur d'accord entre modèle et données.

Les résultats ainsi obtenus sont indiqués dans le tableau $\mathrm{V}$. 
Tableau V. Résidus quadratiques moyens résultant de l'ajustement de chacun des sous-modèles aux données belges.

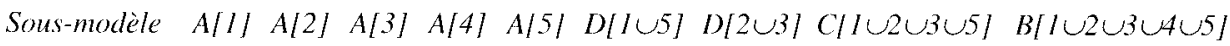

Résidu quadr

moyen $(\mathrm{m})$

$1,06 \quad 0,85 \quad 0,74 \quad 0,58 \quad 0,63$

0,97

0,76

0,90

0,75

Les valeurs des paramètres a, c, d, r, p, m étant ici déjà fixécs (voir tableau II), l'ajustement ne porte que sur le paramètre $b_{i}$, qui explique le niveau de chaque courbc helge dans le faisceau défini par ces valeurs de a, c, d, r, p, m.

Au vu de ces résultats, il est clair que c'est le sous-modèle A[4], établi sur la région 4 , qui convient le mieux aux données belges de croissance en hauteur dominante : ceci confirme l'impression visuelle provoquée par l'apparence tendue des courbes belges. Vient ensuite le sous-modèle $\mathrm{A}[5]$, ce qui est à première vue surprenant ; mais ceci s'explique par le fait que sa forme, tout en étant un peu plus concave, est assez proche de celle de A[4] dans la gamme de fertilité étroite et plutôt médiocre couverte par les données belges, alors qu'elle en diffère nettement dans les fertilités plus élevées. Les autres sous-modèles sont nettement moins satisfaisants, jusqu'à $\mathrm{A}[1]$ qui est le pire de tous en raison de sa forte concavité.

\section{DISCUSSION ET CONCLUSION}

\section{Variabilité intra-régionale de la forme de la croissance}

Nous avons vu, en examinant la figure 2, qu'elle n'est pas négligeable : il y a, dans chaque région, des croisements de courbes assez nombreux. Les résidus quadratiques moyens intra-région donnés dans le tableau III pour le modèle A quantifient bien cette variabilité ; ils sont ici supérieurs à la valeur de $0,50 \mathrm{~m}$ à laquelle on peut s'attendre en ajustant notre modèle mathématique à un faisceau de courbes observées bien étagées (comme pour les plantations d'une espèce résineuse ou les taillis simples d'une espèce feuillue dans une région, par exemple).

Cette variabilité n'est pas uniforme en fonction de l'âge ; si on examine l'écart type des résidus à âge donné du modèle A pour les peuplements d'une région, on constate qu'il passe par un maximum vers $20-50$ ans selon les régions, puis par un minimum vers $80-110$ ans, avant de remonter à un niveau inférieur au premier maximum ; ceci correspond bien à l'impression visuelle fournie par la figure 4 qui montre les dix courbes de résidus en fonction de l'âge de la région 1 .

Dans la pratique, cette variabilité assez forte dans le jeune âge conduit, quel que soit le modèle retenu, à éviter autant que possible les estimations de niveau de fertilité à partir de l'observation de l'âge et de la hauteur dominante de peuplements de moins de 50 ans. En revanche, c'est autour de 100 ans que cette estimation sera le plus robuste. Cette conclusion est indépendante de l'âge de référence choisi pour expliciter le niveau de fertilité $b_{i}$ en termes de hauteur dominante atteinte à cet âge de référence : elle dépend plutôt de l'étendue des âges présent dans les données, l'âge de variance minimum se situant aux environs du milieu de cette étendue.

\section{Variabilité inter-régionale de la forme de la croissance}

L'examen des résidus du modèle d'ensemble $\mathrm{B}$ a montré que celui-ci ne décrit pas 
correctement la forme de la croissance dans la région 4 , alors qu'on peut admettre que les régions $1,2,3$ et 5 sont correctement représentées par un même sous-modèle dans le modèle $\mathrm{C}$. La forme de la croissance de la région 4 , telle qu'elle peut être observée sur notre échantillon, diffère significativement de celle des autres régions.

$\mathrm{Vu}$ la large gamme de fertilités échantillonnée dans chaque région, il semble difficile d'attribuer cette différence à telle ou telle caractéristique du sol ; nous n'avons toutefois pas les moyens de rejeter définitivement cette hypothèse, car nous ne disposons pas d'analyses fines du sol dans les 50 peuplements étudiés.

Une autre hypothèse pourrait être celle d'une sylviculture particulière appliquée aux jeunes peuplements de chêne sessile de la région 4 au XIX ${ }^{\mathrm{e}}$ siècle; nous ne pouvons pas la rejeter, mais nous n'avons pas trouvé d'indice en ce sens dans la tradition orale ou dans les documents existants.

On pourrait aussi penser à l'expression d'une différence génétique. Les études en cours en France sur la structuration de la diversité génétique du chêne sessile, par analyse des isoenzymes et de l'ADN chloroplastique (Petit et al 1993, Zanetto et Kremer 1995), fourniront peut-être ultérieurement des éléments de réponse à cette question.

On pourrait enfin penser à l'expression d'une différence climatique. Cette hypothèse est confortée par l'étude réalisée dans le cadre du même programme par Gilbert et Franc (1995) sur le climat dans le Nord de la France. Ils y mettent en évidence que la région 4 se caractérise à la fois par une pluviométrie faible (de l'ordre de $600 \mathrm{~mm}$ ) avec un creux assez net d'avril à août et par une évapo-transpiration potentielle assez forte : ceci conduit à un déficit hydrique (calculé pour une capacité de stockage de $100 \mathrm{~mm}$ d'eau dans le sol) plus élevé que dans les autres régions. Cette interprétation séduisante demanderait à être confirmée par l'observation de la croissance d'autres peuplements dans le triangle Angers-Poitiers-Paris, repéré dans cette étude comme sujet à un déficit hydrique relativement élevé. Elle semble contredite par l'observation des courbes belges qui présentent apparemment la même forme que celles de la région 4, alors qu'elles proviennent d'une région sans déficit hydrique (pluviométrie supérieure à $1100 \mathrm{~mm}$ )...; mais cette contradiction clle-même n'est pas sûre, à cause de l'étroitesse du domaine de fertilité couvert par l'échantillon belge et de l'incertitude qui en résulte sur la forme de sa croissance : il faudrait le compléter avec des peuplements de bonne fertilité.

La figure 7 montre la forme des trois modèles : modèle $B$ correspondant à l'ensemble des régions, sous-modèle $C[1 \cup 2 \cup 3 \cup 5]$ pour les quatre régions de forme semblable, sous-modèle C $|4|=\mathrm{A}[4]$ pour la région 4.

Nous proposons d'adopter le modèle d'ensemble B pour la modélisation de la croissance du chêne sessile en France, en général. On s'expose ainsi à un biais pouvant aller jusqu'à 1 m dans la région 4 (et un peu moins dans la région 1 ), dans un sens ou dans l'autre selon l'âge et la région. Pour une modélisation plus régionale, on peut utiliser le modèle C, c'est-à-dire le sousmodèle $C[1 \cup 2 \cup 3 \cup 5]$ ou $C[4]$ selon la région visée ; les biais risqués sont alors inférieurs à $0,50 \mathrm{~m}$ (voir figure 5) et non significatifs. Il sera donc généralement inutile de penser à une modélisation plus fine, basée sur les modèles D ou A.

Les expressions des modèles retenus, avec $\mathrm{H}_{100}=$ hauteur dominante à 100 ans comme indice de fertilité, sont les suivantes :

Modèle B, pour l'ensemble des 5 régions :

$$
\begin{aligned}
\mathrm{H} & =0,30+(-0,039339 * \mathrm{~A}+0,78595 \\
& \left.* \mathrm{H}_{100}-7,10703\right) * \mathrm{I}-\exp (-0,0024168 \\
& \left.\left.* \mathrm{~A}^{1.56660)}\right)\right]^{0,71192} \\
& +\left(0,10445+0,0023513 * \mathrm{H}_{1(0)}\right) * \mathrm{~A}
\end{aligned}
$$



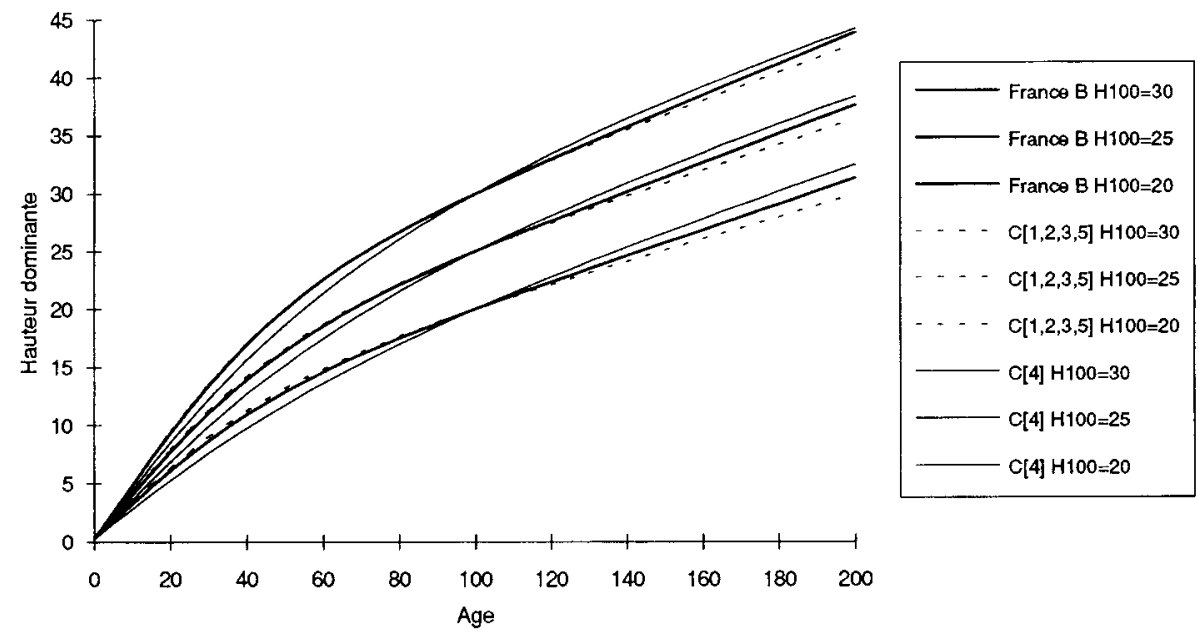

Fig 7. Dessin des courbes du modèle B (ensemble des régions), et des deux sous-modèles du modèle $C$ (régions $1 \cup 2 \cup 3 \cup 5$, et région 4), pour trois valeurs de l'indice de fertilité $\mathrm{H}_{100}=20,25,30$ mètre (abscisse : âge dominant compté à partir de $0,30 \mathrm{~m}$ en années, ordonnée : hauteur dominante en mètre).

Sous-modèle $C[1 \cup 2 \cup 3 \cup 5]$, pour les régions $1,2,3,5$ :

$$
\begin{aligned}
\mathrm{H} & =0,30+(-0,058286 * \mathrm{~A}+0,81311 \\
& \left.* \mathrm{H}_{100}-5,88105\right) *[1-\exp (-0,0124623 \\
& \left.\left.* \mathrm{~A}^{1,17144}\right)\right] 1,04867 \\
& +\left(0,10622+0,0024161 * \mathrm{H}_{100}\right) * \mathrm{~A}
\end{aligned}
$$

Sous-modèle $\mathrm{C}[4]=\mathrm{A}[4]$, pour la région 4:

$$
\begin{aligned}
\mathrm{H} & =0,30+\left(0,005985 * \mathrm{~A}+1,20318 * \mathrm{H}_{100}\right. \\
& -13,36828) *[1-\exp (-0,0186715 \\
& \left.\left.* \mathrm{~A}^{1,00328}\right)\right]^{1,13625}+0,103133 * \mathrm{~A}
\end{aligned}
$$

où $\mathrm{A}$ est l'âge dominant, compté à partir du moment où la hauteur dominante atteint $0,30 \mathrm{~m} ; \mathrm{H}$ est la hauteur dominante à l'âge A; $\mathrm{H}_{100}$ est l'indice de fertilité : hauteur dominante atteinte pour $\mathrm{A}=100$; $\exp ($ ) est la fonction exponentielle

\section{Effet de la date}

En étudiant l'effet de la date sur les résidus du modèle le plus fin, nous avons constaté un effet synchrone dans toutes les régions, correspondant à une remontée de la croissance observée par rapport aux modèles depuis 1955-60. Cet effet n'est important que dans les régions 3 et 4 , où les courbes observées passent en moyenne de $0,40 \mathrm{~m}$ en-dessous de leur modèle en 1958 à 0,50 m au-dessus de leur modèle en 1993. On retrouve d'ailleurs de tels écarts à des dates plus anciennes dans certaines régions, mais sans synchronisation entre régions (fig 6).

À quoi peut-on attribuer ce gain de croissance apparent, de l'ordre de $1 \mathrm{~m}$ dans les régions 3 et 4, de l'ordre de $0,40 \mathrm{~m}$ dans les autres ? La première idée qui vient à l'esprit, en ces temps industriels, est de le lier à une «amélioration» récente des conditions de milieu (dépôts azotés, $\mathrm{CO}_{2}$ dans l'atmosphère...)

On peut aussi proposer une autre explication partielle. Lors de la réalisation des mesures sur les arbres abattus, on voit clairement que certains houppiers ont connu un dépérissement sommital suivi d'une reprise active de la croissance à partir d'un bour- 


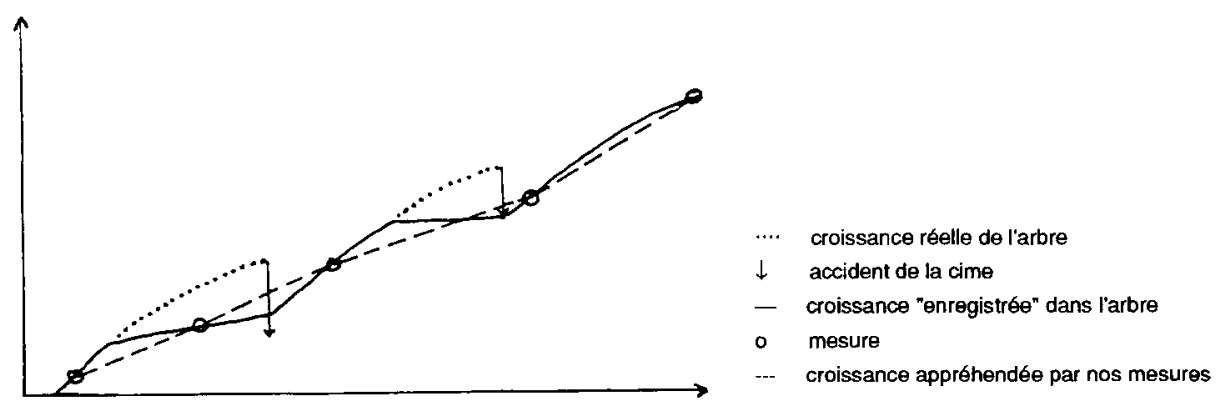

Fig 8. Schéma possible de la croissance en hauteur du chêne sessile et de l'appréhension que nous en avons par nos mesures d'analyse de tige (temps en abscisse, hauteur en ordonnée).

geon ou d'une branche restée vivante. Il n'est pas impossible que la croissance normale des chênes, surtout quand ils sont âgés, suive un tel rythme irrégulier, marqué par les étapes : accident et dépérissement de la partie supérieure - relais par un niveau inférieur - croissance - accident... Si le début du dernier intervalle mesuré sur un arbre est proche du dernier accident, on peut alors observer un relèvement final de la courbe qui n'est en fait qu'un artefact de la méthode de mesure, selon le schéma de la figure 8 .

Quelles que soient les causes profondes de cet effet des années récentes, nous voulons avoir une idée de son impact éventuel sur la forme et le niveau des courbes de croissance. Pour cela, nous avons ré-ajusté le modèle mathématique MM sur l'ensemble des courbes observées, après avoir enlevé à chacune les 35 dernières années (1959-1993). Le résultat en est le modèle ajusté B', caractérisé par les valeurs suivantes des paramètres :

$\mathrm{a}=-0,0315005$

$\mathrm{c}=46,25123$

$\mathrm{d}=1.56676$

$r=0,703775$

$\mathrm{p}=0,128799$

$\mathrm{m}=0,00180112$
La forme de ce modèle est presque identique à celle du modèle B (figure 9), sauf une très légère diminution de la pente audelà de 130 ans dans les meilleures fertilités.

Nous avons aussi comparé les niveaux fournis par les deux ajustements B et B' pour chaque courbe observée, après les avoir exprimés, dans chaque modèle, en termes de hauteur dominante à 100 ans, $\mathrm{H}_{100}$ : pour une courbe donnée, les deux valeurs sont bien sûr différentes, jusqu'à $\pm 1,5 \mathrm{~m}$, à cause de la perte des 35 dernières années dans le deuxième modèle. Mais la moyenne de ces différences $\left(\mathrm{H}_{100}-\mathrm{H}_{100}\right)$ a une valeur de $-0,06 \mathrm{~m}$, un écart-type de $0,08 \mathrm{~m}$, et ne diffère pas significativement de zéro.

Globalement, la suppression des 35 dernières années dans les données observées ne change donc pratiquement rien à la forme des courbes ni à leur niveau : l'impact de l'effet date constaté (relèvement final des courbes) sur les résultats de la modélisation est actuellement négligeable. Mais il s'agit d'une modélisation de la croissance passée : il n'en serait peut-être pas de même, du moins en ce qui concerne le niveau, si l'on comparait des croissances juvéniles récentes et anciennes sur la même station. 


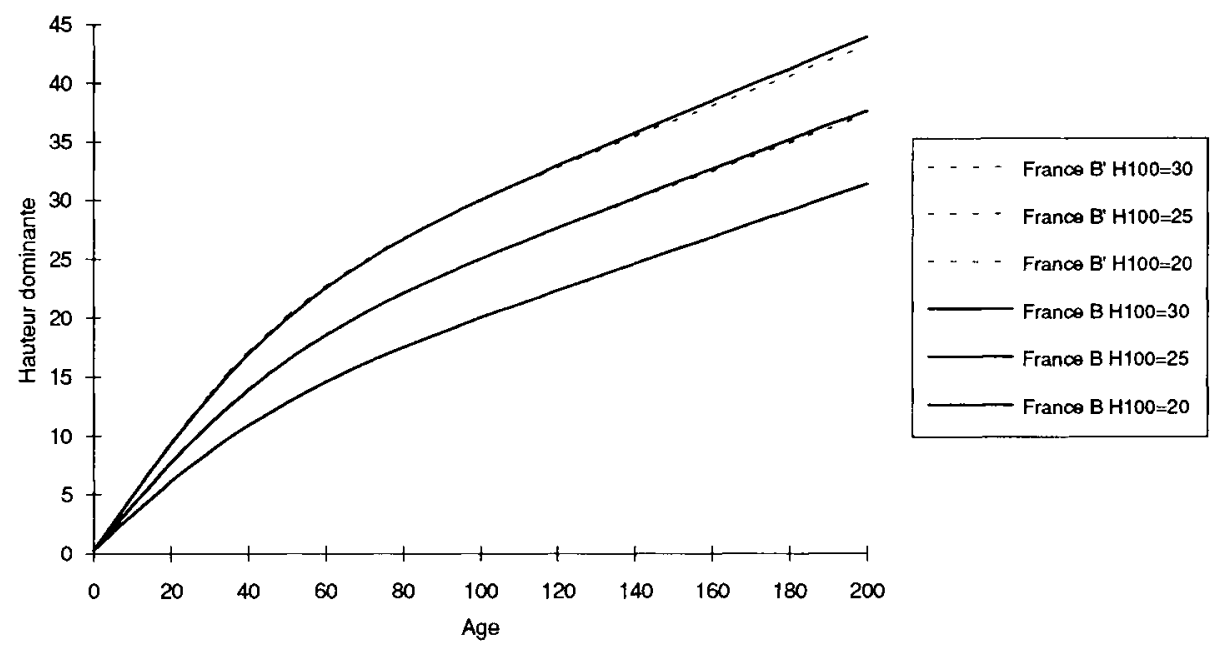

Fig 9. Dessin des courbes du modèle B (ajusté sur les données complètes), et du modèle B' (ajusté sur les données sans les 35 dernières années), pour trois valeurs de l'indice de fertilité $\mathrm{H}_{100}=20,25$, $30 \mathrm{~m}$ (abscisse : âge dominant compté à partir de $0,30 \mathrm{~m}$ en années, ordonnée : hauteur dominante en $\mathrm{m})$.

\section{Comparaison avec des modèles existants}

Nous disposons des éléments de comparaison suivants :

- les tables de production britanniques de Hamilton et Christie (Forestry Commission 1981);

- les tables de production pour le BadenWürttemberg, proche de la France (Zimmerle 1930);

- la table de production de Pardé, avec une seule classe de fertilité, pour la région de la Loire en France (Pardé 1962);

- les tables de production de Jüttner pour la Prusse et la Basse-Saxe (Schober 1975);

- le modèle de production pour les jeunes peuplements de chênes en Suisse (Schütz et Badoux 1979).

En fait, les deux demiers se prêtent mal à une comparaison. Les tables de Jütner (Schober) sont établies dans des régions éloignées de la France et ne donnent pas l'évolution de la hauteur dominante, mais seulement celle de la hauteur moyenne (évolution d'ailleurs très proche, pour la meilleure classe de fertilité, de celle de la hauteur dominante chez Pardé). Le modèle de Schütz et Badoux ne concerne que des peuplements âgés de moins de 60 ans. La figure 10 montre donc les trois autres modèles, en comparaison avec notre modèle d'ensemble B.

Le modèle britannique de Hamilton et Christie présente la croissance la moins soutenue : sa pente chute très rapidement et continuement à partir de 70 ans. Elle est un peu plus soutenue dans le modèle de Zimmerle, qui ressemble au nôtre jusque vers 100 ans. Elle l'est encore un peu plus dans le modèle de Pardé. Mais elle l'est beaucoup plus dans notre modèle, où l'accroissement en hauteur est quasiment constant après 100 ans, comme dans les données utilisées pour son ajustement (cf. fig 2).

On peut proposer à cela des explications partielles: 


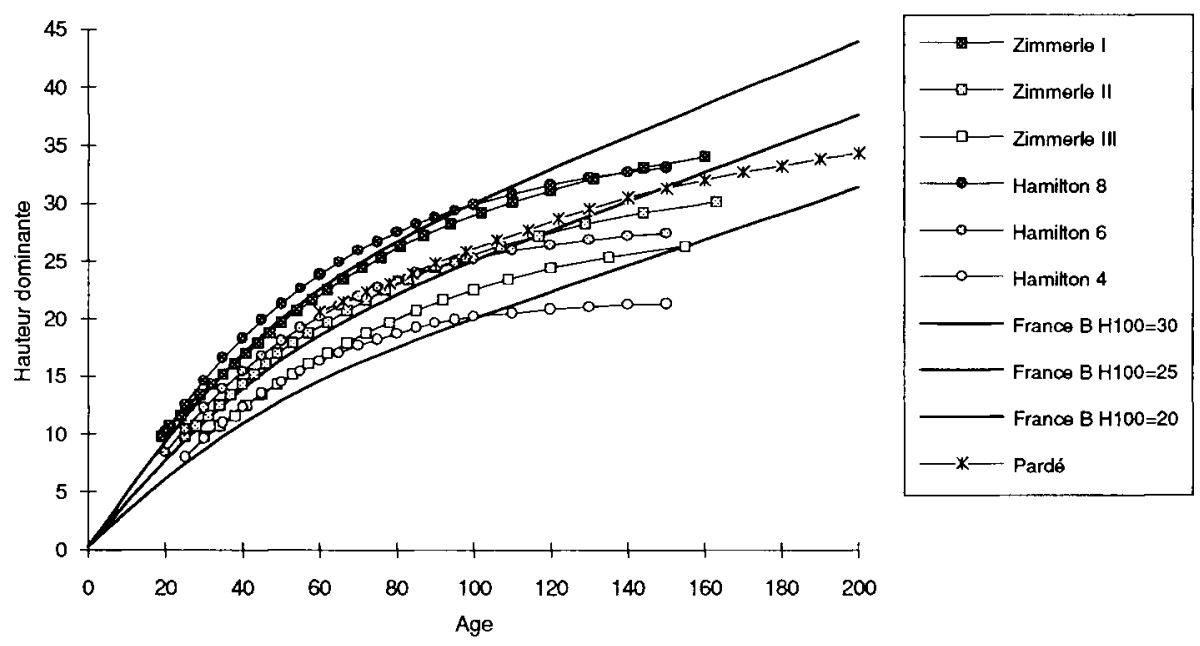

Fig 10. Comparaison entre notre modèle général B et d autres modèles décrivant la croissance en hauteur dominante du chêne : Zimmerle (Baden-Württemberğ). Hamilton and Christie (Great-Britain), Pardé (France) (abscisse : âge en années, ordonnée : hauteur dominante en m).

- le modèle de Hamilton et Christie est probablement construit à partir de placettes permanentes pas très âgées ( 100 ans ?) et composées très majoritairement de Quercus robur planté, dont la forme de la croissance peut être différente de celle de Quercus petraea:

-Zimmerle se déclare «obligé d'arrêter ses tables à 160 ans, par manque de placettes âgées »; on peut penser qu'il en a jusque vers 150 ans; il dit aussi que $63 \%$ de ses placettes sont constituées de Quercus robur;

- pour Pardé, il s'agit bien de Quercus petraea, mais 2 ou 3 placettes seulement dépassent 160 ans.

Nous ignorons d'autre part quelles furent les méthodes utilisées par les auteurs pour modéliser leurs données. En tous cas, ces différences ne sont pas dues à une éventuelle accélération récente de la croissance, puisque nous obtenons le même résultat en arrêtant nos observations à 1958.
Nos données sont concrètes et robustes, la moitié des croissances reconstituées dépassent 150 ans et le quart 175 ans. On a vu que l'éventuel effet de la date (période récente) avait un impact négligeable sur les résultats de la modélisation. On peut toutefois laire une objection à la méthode : l'utilisation de ces données est basée sur l'hypothèse que la croissance passée des 100 arbres/hectare actuellement les plus gros représente bien l'histoire de la croissance en hauteur dominante du peuplement ; cette hypothèse peut être critiquée et il y a là matière à une intéressante étude méthodologique, mais il semble peu probable qu'elle puisse conduire à une correction de nos résultats d'une ampleur telle qu'ils rejoindraient les résultats allemands ou, a fortiori, britanniques.

En définitive, nous pensons que beaucoup de modèles actuellement publiés et utilisés sous-estiment la croissance en hauteur dominante des peuplements âgés, et ceci ne concerne pas que les chênes. 
Remerciements : Ce travail a été partiellement subventionné par la Communauté européenne, dans le cadre du programme de recherche AIR-CT92-0608 ( $P$ Production of quality wood from broadleaves $»$ ).

\section{RÉFÉRENCES}

Duplat P, Tran-Ha Mien (1990) Modélisation d'un faisceau de courbes de croissance en hauteur : ajustement instantané d'un modèle à $p$ paramètres communs à toutes les courbes et 1 paramètre libre. $I n$ : Research in Forest Inventory, Monitoring Growth and Yield (World congress IUFRO, Montréal, 1990, joint sessions of $\$ 4.02$ et $\$ 4.01$ ), Publication FWS3-90, Virginia Polytechnic Institute and State University, Blacksburg, VA, États-Unis

Forestry Commission (1981) Yield Models for Forest Management. Forestry commission booklet 48 . Alice Holt Lodge, 1981, R44 a-c

Gilbert JM, Franc A (1995) Relationships between top height growth of Quercus petraea and climatic variability in the northern half of France. Final report, CEE-AIR Project contract CT92-0608

Matérn B (1976) On estimating the dominant height. Särtryck ur Sveriges Skogvärdsförbunds Tidskrift $74,51-53$
Pardé $\mathbf{J}$ (1962) Table de production pour les forêts de chêne rouvre de qualité tranchage du secteur ligérien. Note technique $\mathrm{n}^{\circ} 11$, mars 1962,13 p. Station de recherches forestières, Inra, Nancy, France.

Petit RJ, Kremer A, Wagner DB (1993) Geographic structure of chloroplast DNA polymorphisms in European oaks. Theor Appl Genet 87, 122-128

Seber GAF, Wild CJ (1989) Nonlinear Regression. John Wiley and Sons, New York

Schmidt R (1982) Advances in Non Linear Parameter Optimization. Lecture notes in control and information sciences $n^{\circ} 37$, Springer-Verlag, Berlin

Schütz JP, Badoux E (1979) Production de jeunes peuplements de chênes en relation avec la station. Mémoires de l'Institut fédéral de recherches forestières, vol 55, fasc 1, Birmensdorf, Suisse

Schober R (1975) Ertragstafeln wichtiger Batmarten. JD Sauerländer's Verlag, Francfort-sur-le-Main, Allemagne

Zanetto A, Kremer A (1995) Geographical structure of gene diversity in Quercus petraea (Matt) Liebl. I Monolocus patterns of variation. Heredity 75 , 506-517

Zimmerle (1930) Hilfszahlen zur Boniticrung, Vorrats- und Zuwachsschätzung in reinen Eichenbeständen. In: Mitteilungen der Wiurttembergischen Forstlichen Versuchsanstalt 1930, Stuttgart 